\title{
MedienPädagogik
}

Zeitschrift für Theorie und Praxis der Medienbildung

www.medienpaed.com

ISSN 1424-3636

Themenheft Nr. 37: Medienpädagogik als Schlüsseldisziplin in einer mediatisierten Welt. Perspektiven aus Theorie, Empirie und Praxis Herausgegeben von Henrike Friedrichs-Liesenkötter, Lara Gerhardts, Anna-Maria Kamin und Sonja Kröger

\section{Schulische Medienbildung im Spannungsfeld von Medienpädagogik und Fachdidaktik}

\author{
Bardo Herzig und Alexander Martin
}

\section{Zusammenfassung}

Die zunehmende Digitalisierung und Mediatisierung der Lebenswelt sind auf gesellschaftlicher und individueller Ebene mit grundlegenden und unausweichlichen Wandlungsprozessen verbunden. Somit stellt sich die Frage, welche Aufgaben Erziehung und Bildung unter diesen Rahmenbedingungen zukommen. Bildungspolitisch besteht Konsens darüber, dass Schulen bei der Vermittlung von Medienkompetenz eine wichtige Rolle spielen (müssen). Die konkrete Umsetzung soll dabei nicht in einem isolierten Lernbereich, sondern in der Verantwortung aller (Unterrichts-)Fächer liegen. Setzt man bildungspolitisch auf eine integrative Realisierung von Medienbildung, d.h. ohne einen eigenen Lernbereich oder ein eigenes Fach, so bedeutet dies, dass entsprechend abgestimmte schulische Lerngelegenheiten geschaffen werden müssen, die in der Summe der Beiträge aller Unterrichtsfächer einen umfassenden Kompetenzerwerb ermöglichen. Rahmenbedingungen, Vorgaben und Voraussetzungen für eine erfolgreiche Bewältigung dieser Aufgabe werden im Beitrag sowohl aus medienpädagogischer als auch aus fachdidaktischer Perspektive aufgegriffen und mit Blick auf Chancen und Herausforderungen beleuchtet. 


\title{
Media Literacy in School Between the Poles of Media Pedagogy and Subject Didactics
}

\begin{abstract}
The ongoing and increasing digitalization and mediatization of the lifeworld are leading to fundamental and inevitable change processes on a social and individual level. This raises the question of which tasks are coming up to educational processes. In terms of educational policy, there is agreement that schools (have to) play an important role in teaching media literacy. The concrete implementation is not meant to take place within one separate learning area but rather as part of all school subjects. This article discusses which problems and challenges arise for teaching media literacy in school between the poles of Media Pedagogy and Subject Didactics. If there is no subject or special learning area for imparting media literacy, then there must be enough learning opportunities geared to all subjects for fostering broad acquisition of media-related competencies. In this article, the preconditions and requirements for a successful accomplishment of this task will be taken up from the point of view of both Media Pedagogy and Subject Didactics.
\end{abstract}

\section{Ausgangslage}

Die Entwicklung der Medien bzw. der Medienlandschaft vom Buchdruck bis zum Internet hat immer wieder Diskussionen darüber ausgelöst, welche Chancen und Problemlagen mit diesen Entwicklungen verbunden sind und wie diese (medien-)pädagogisch bearbeitet werden können. Das Verhältnis von Mensch und Medium wurde dabei von unmündigen und dem Medienangebot hilflos ausgelieferten Rezipierenden bis zu entwicklungsfähigen und kompetent Medienhandelnden ausgedeutet. Entsprechend unterschiedlich stellten sich (medien-)pädagogische Handlungsempfehlungen dar.

Mit der Digitalisierung ist ein Prozess angestossen worden, dessen Ausmass und Bedeutsamkeit mit bisherigen Entwicklungen nicht vergleichbar ist. Begriffe wie〈Disruption〉 oder〈Megatrend〉 versuchen dem Umstand Rechnung zu tragen, dass sich mit der Digitalisierung und der 
damit verbundenen zunehmenden Mediatisierung die Lebenswelt in allen Lebensbereichen - Alltag, Freizeit, Beruf - grundlegend und nachhaltig wandelt und dieser Wandel für den Einzelnen im Grundsatz keine〈Ausweichoption〉 mehr zulässt: die Digitalisierung der Lebenswelt mit ihren Auswirkungen auf die individuelle und gemeinschaftliche Lebensführung ist nicht abwendbar. Damit stellt sich umso dringlicher die Frage, welche Aufgaben Erziehung und Bildung unter diesen Rahmenbedingungen zukommen.

In ihrer Strategie zur Bildung in der digitalen Welt hat die Kultusministerkonferenz verbindliche Anforderungen formuliert, «über welche Kenntnisse, Kompetenzen und Fähigkeiten Schülerinnen und Schüler am Ende ihrer Pflichtschulzeit verfügen sollen, damit sie zu einem selbstständigen und mündigen Leben in einer digitalen Welt befähigt werden» (KMK [Kultusministerkonferenz] 2016, 11). Eine Umsetzung dieser Aufgabe sieht die KMK nicht in einem isolierten Lernbereich, sondern in der Verantwortung aller (Unterrichts-)Fächer (ebd., 12).

Im Folgenden wird diskutiert, welche Herausforderungen und Problemlagen sich für eine schulische Medienbildung unter den genannten Bedingungen im Spannungsfeld von Medienbildung und unterrichtsfachbezogener Bildung bzw. von Medienpädagogik und Fachdidaktik ergeben und welche Aufgaben damit verbunden sind.

\section{Medienpädagogik und schulische Medienbildung}

Die Medienpädagogik versteht sich als Reflexions- und Handlungs- bzw. Gestaltungswissenschaft, die auf eine «forschungsbasierte sowie praxisrelevante Theoriebildung im Hinblick auf erziehungs- und bildungsrelevantes Handeln in Medienzusammenhängen» zielt (Sektion Medienpädagogik der Deutschen Gesellschaft für Erziehungswissenschaft - DGfE 2018, 202). Um dieses Ziel einer reflexiven Absicherung und einer wissenschaftlich fundierten Begründung medienpädagogischen Handelns zu erreichen, muss sie

- spezifische Begriffsklärungen vornehmen,

- medienpädagogisch relevante Zustände beschreiben und überprüfen, 
- Zielvorstellungen für pädagogisch-medienbezogene Aktivitäten formulieren und begründen sowie

- Hypothesen zum Zusammenhang zwischen Voraussetzungen, Zielen und Vorgehensweisen medienpädagogischen Handelns formulieren und prüfen (Tulodziecki, Herzig, und Grafe 2019, 45).

Zentraler Gegenstand der Medienpädagogik sind Medien, deren terminologische Bestimmung eine der genannten Aufgaben ist. Dabei erweist es sich aus verschiedenen Gründen als zweckmässig, Medien als «Mittler [zu verstehen], durch die in kommunikativen Zusammenhängen potenzielle Zeichen mit technischer Unterstützung aufgenommen bzw. erzeugt und verarbeitet, übertragen, gespeichert oder wiedergegeben bzw. präsentiert werden und verfügbar sind» (ebd., 33). Unter einen solchen Medienbegriff lassen sich sowohl verschiedene Medienarten und mediale Angebote als auch die mit der Erstellung und Verbreitung verbundenen Infrastrukturen einordnen.

Unterstellt man, dass die Medieninhalte in Wechselwirkung zwischen Individuum und Gesellschaft entstehen, in Zusammenhang mit ihrer Form Einflüsse auf Individuum und Gesellschaft ausüben und mit ökonomischen, rechtlichen, personalen, politischen oder institutionellen Rahmenbedingungen ihrer Produktion und Verbreitung verflochten sind, wird deutlich, dass die Medienpädagogik Anschlussstellen zu weiteren Disziplinen aufweist wie der Medien- und Kommunikationswissenschaft, der Soziologie, der Politikwissenschaft, der Semiotik oder der Informatik. Mit ihrer grundständigen Verankerung in der Erziehungswissenschaft referenziert sie darüber hinaus auf weitere Bezugsdisziplinen wie z.B. die Psychologie oder die Philosophie (Tulodziecki, Herzig, und Grafe 2019, 39).

Historisch gesehen hat sich die Medienpädagogik in zwei Akzentsetzungen entwickelt: Zum einen geht es um die Frage, wie Medien in Lehrund Lernprozessen genutzt werden können, um spezifische Lernaktivitäten anzuregen und zu unterstützen. Dieser Zweig hat sich in Form der Mediendidaktik als Wissenschaft und Lehre vom Lehren und Lernen mit Medien etabliert (z.B. Tulodziecki und Herzig 2009; Kerres 2018). Zum anderen geht es um Erziehungs- und Bildungsaufgaben, die sich angesichts der zunehmenden Mediennutzung durch Kinder und Jugendliche stellen, 
und um ihre Bewältigung. Während anfänglich der Erziehungsgedanke vorherrschend war, ist mit der zunehmenden Betonung von Mündigkeit, Selbstbestimmung, Kritikfähigkeit und Reflexivität als Zielvorstellungen der Erziehungsbegriff stärker an den Bildungsbegriff herangerückt und beide finden in der Wissenschaft und Lehre von medienbezogenen Erziehungs- und Bildungsaufgaben ihren Niederschlag.

Medienpädagogik als Wissenschaft entfaltet ihre Praxis als Lehren und Lernen mit Medien und als Medienerziehung und Medienbildung, wobei das Verhältnis wechselseitig in dem Sinne ist, dass Wissenschaft und Praxis gemeinsam an praxisbezogener Theoriebildung und reflektierter Praxis arbeiten. Der Bezug der Medienpädagogik zur Schule liegt bei der Mediendidaktik auf der Hand. Als Institution des Lehrens und Lernens nimmt die Frage, wie Unterrichtsmittel in Form von Medien Lernprozesse anregen und unterstützen können, seit langem breiten Raum ein. Erste Überlegungen finden sich schon bei Johan Amos Comenius (1658). In der Allgemeinen Didaktik führte Heimann (1962) Medien als eigenes Strukturelement von Unterricht ein. Neben der grundsätzlichen Bedeutung der Medien für Lehr-Lernprozesse liegt es nahe, dass spezifische Fragen der Gestaltung von Unterricht mit Hilfe von Medien fachdidaktisch diskutiert werden und es im Eigeninteresse der Unterrichtsfächer liegt, entsprechende Möglichkeiten unter den jeweiligen medialen Bedingungen auszuloten (s.u.). Ansätze einer Medienerziehung hingegen wurden zunächst in Bezug auf einzelne Medien(-arten) entwickelt, z.B. als Film- oder Fernseherziehung, bevor im Zuge der Diskussion um eine grundlegende Medienbildung auf medienartübergreifende Fähigkeiten, Fertigkeiten und Einstellungen abgehoben wurde. Anders als in der Mediendidaktik waren diese Aufgaben nicht genuin mit jedem Unterrichtsfach verbunden, sondern einzelne Fächer wurden im Sinne von Leitfächern als prädestiniert für eine Auseinandersetzung betrachtet, u.a. die Fächer Deutsch, Kunst, Musik oder weitere gesellschaftswissenschaftliche Fächer (KMK 1995, 20). Vorstellungen dazu, welche Kompetenzen in der Schule aus Sicht der Medienpädagogik entwickelt werden sollten, finden sich z.B. in einem fächerübergreifenden Orientierungsrahmen schulischer Medienbildung (Tulodziecki, Herzig, und Grafe 2019, 208). 
Die in der Strategie der KMK zur Bildung in der digitalen Welt (2016) festgelegte Umsetzung der Medienbildung in der Schule setzt auf die Beteiligung aller Fächer und kann auf zwei Vorgehensweisen beruhen: zum einen können, von dem übergreifend formulierten Kompetenzrahmen für die Medienbildung ausgehend, einzelne Aufgaben der Medienbildung den Unterrichtsfächern zugeordnet werden; zum anderen kann, vom Medienbezug des jeweiligen Unterrichtsfaches ausgehend, danach gefragt werden, welchen Beitrag das Fach zu übergreifenden Zielen der Medienbildung leisten kann (Spanhel 1999, 32). Beide Strategien verweisen auf spezifische Herausforderungen und Problemlagen einer schulischen Medienbildung:

- Medienpädagogische Aufgaben werden nicht von einem entsprechenden Fach- oder Lernbereich übernommen, sondern 〈aufgelöst) und auf Unterrichtsfächer verwiesen, die nicht alle unmittelbar Bezugsdisziplinen darstellen. Dies bedeutet, dass die Medienpädagogik nur den Referenzrahmen bildet, der durch Beiträge der Fächer inhaltlich ausgestaltet werden muss. Ist der Kompetenzrahmen darüber hinaus nicht medienpädagogisch disziplinär verankert und begründet, sondern Ergebnis bildungspolitischer Konsensbildung, besteht die Gefahr von 〈Leerstellen〉, d.h. einer ggf. eklektischen Anlage von Medienbildung.

- Wenn medienpädagogische Expertise nicht über einen eigenen Lernbereich in schulische Medienbildungsprozesse eingebracht wird, dann muss sie in die einzelnen Fächer Eingang finden. Dies bedeutet, dass zum einen alle Fachlehrpläne um entsprechende medienbezogene Kompetenzen erweitert werden müssen und zum anderen in der hochschulischen Ausbildung entsprechende medienpädagogische Kompetenzen insbesondere in den Fachdidaktiken aufgebaut werden müssen.

- Die schulische Umsetzung von Medienbildung erfordert einen komplexen Schulentwicklungsprozess, in dem ein schulspezifisches Medienbildungscurriculum über die Jahrgangsstufen und Fächer hinweg als konsistentes Konstrukt entwickelt werden muss. Ziel ist dabei, für Schülerinnen und Schüler abgestimmte Lerngelegenheiten zu schaffen, in denen die avisierten Kompetenzen entwickelt werden können. 
Exemplarisch lassen sich die genannten Aspekte an dem Versuch illustrieren, zu Beginn der 1980er Jahre eine informationstechnische Grundbildung - zunächst als eigenständigen Bereich, später integrativ zur $\mathrm{Me}$ dienbildung - einzuführen. Dieser Versuch muss letztlich als gescheitert angesehen werden, insbesondere aus Gründen einer fehlenden Konkretisierung des Inhalts- und Gegenstandsbereiches der Grundbildung und dessen Abstimmung mit und Abgrenzung von den beteiligten Fächern (Herzig 2012, 1:34-42; Wilkens 2000).

Vor dem Hintergrund dieser Überlegungen stellt sich die Frage, wie der Anspruch einer umfassenden Medienbildung im Sinne des Kompetenzrahmens der KMK eingelöst werden kann. Eine Schlüsselrolle dürfte hier den Fachdidaktiken zukommen. Insbesondere für die Fächer, die keine Bezugsdisziplinen der Medienbildung darstellen und die bisher in der Medienbildung keine Leitfunktion übernommen haben, stellt sich die Aufgabe, Bezüge des betreffenden Faches zur digital vernetzten Welt aufzuarbeiten und zu prüfen, welche Beiträge es zum Kompetenzerwerb von Schülerinnen und Schülern leisten kann.

\section{Fachdidaktik und schulische Medienbildung}

Wenngleich Fachdidaktiken enge Bezüge zu Nachbardisziplinen aufweisen und innerhalb ihrer Aussagesysteme auf interdisziplinäre Bezugnahmen angewiesen sind, handelt es sich um eigenständige Forschungsdisziplinen und Hochschulfächer. Ausdruck dieser Eigenständigkeit sind auf organisatorischer Ebene etwa eigene Fachgesellschaften, Tagungen und Nachwuchsprogramme und auf inhaltlicher Ebene eigene Tätigkeitsfelder und spezifische Aufgaben und Ziele (Leuders 2015, 215-16). Dieser disziplinäre Status als wissenschaftliche Einrichtung ist vergleichsweise jung. Erst 1998 hat die, heute als Gesellschaft für Fachdidaktik (GFD) benannte, damalige Dachgesellschaft verschiedener Einzelfachdidaktiken einen Konsens über Gegenstandsbereiche und Aufgaben der Fachdidaktik beschlossen und veröffentlicht:

«Fachdidaktik ist die Wissenschaft vom fachspezifischen Lehren und Lernen innerhalb und außerhalb der Schule. In ihren Forschungsarbeiten befasst sie sich mit der Auswahl, Legitimation 
und didaktischen Rekonstruktion von Lerngegenständen, der Festlegung und Begründung von Zielen des Unterrichts, der methodischen Strukturierung von Lernprozessen sowie der angemessenen Berücksichtigung der psychischen und sozialen Ausgangsbedingungen von Lehrenden und Lernenden. Außerdem widmet sie sich der Entwicklung und Evaluation von Lehr- und Lernmaterialien» (KVFF [Konferenz der Vorsitzenden Fachdidaktischer Fachgesellschaften] 1998, 13-14).

Trotz aller laufenden Anpassungen und Aktualisierungen kann dieses Verständnis nach wie vor als gültig angenommen werden (Vollmer 2017, 13). Das Erkenntnisinteresse fachdidaktischer Forschung im Sinne einer eigenständigen wissenschaftlichen Disziplin ist also auf fach- bzw. domänenspezifische Lern- und Lehrprozesse gerichtet. Diese Zielperspektive bedarf multiperspektivischer Zugriffe, z.B. in Anlehnung an die jeweilige Bezugs- bzw. Fachwissenschaft, an die Pädagogische Psychologie und an die Allgemeine Didaktik (Parchmann 2013, 32). Die Aufgabe der Fachdidaktik besteht dann darin, «etwas Neues aus dem Gefüge der verschiedenen Erkenntnisse und Theorien zu entwickeln» (ebd.). Dieses Selbstverständnis bricht mit Vorstellungen von einer Fachdidaktik als blosser «Vermittlungswissenschaft» (Weißeno 2015, 5) für bestimmte fachliche Inhalte und rückt stattdessen die Auffassung in den Vordergrund, dass es sich beim fachlichen Lehren und Lernen immer um einen spezifischen, auf bestimmte Gegenstände gerichteten Prozess handelt. Bezüge zu weiteren für das Verständnis von (Fach-)Unterricht relevanten Aspekten sind dabei selbstverständlich zu berücksichtigen, der Referenzrahmen sind aber die jeweiligen Fachgegenstände. Typische unterrichtliche Gegenstandsbereiche, mit denen sich Fachdidaktiken auseinandersetzen, sind dann etwa die Kompetenzen von Schülerinnen und Schülern und Lehrkräften, die Auswahl fachwissenschaftlicher Gegenstände und Zugänge, die Untersuchung der mit diesen Entscheidungen verbundenen Wirkungen und Effekte auf allen Ebenen des Unterrichts und der daran Beteiligten und der darauf fussende Beitrag zur Theoriebildung (ebd., 7-8). Parallel zu den Bemühungen um eine Profilierung der Fachdidaktiken finden sich jüngst auch vermehrt Vorschläge und Diskussionen zur Erarbeitung und Fundierung einer 
(Allgemeinen Fachdidaktik》, verstanden als «Gesamtkonzept fachlicher Bildung» (Bayrhuber 2017, 253), das Gemeinsamkeiten und Zusammenhänge des fachlichen Lernens in verschiedenen Fächern fasst und systematisiert (Abraham und Rothgangel 2017).

Die Kürze des Abrisses zur fachdidaktischen Profilbildung darf nicht darüber hinwegtäuschen, dass bereits die 〈Auswahl〉 der jeweiligen Bezugs- resp. Fachwissenschaft keineswegs trivial ist. Wenngleich zwar die grobe Zuordnung zuallermeist naheliegend ist, ist die Frage der Gewichtung von Teildisziplinen bereits erheblich diffiziler und nicht selten sind im Fachdiskurs selber Rolle und Stellung von Teildisziplinen unklar. Aufgrund der zumeist auch bereits institutionell (Fachdidaktiken sind an Universitäten in der Regel Fächern zugeordnet) noch grösseren Distanz ist die Justierung des Verhältnisses von Fachdidaktiken und benachbarten Bezugsdisziplinen, die ja wiederum auch oftmals eine Vielzahl von Teildisziplinen aufweisen, nicht minder aufwändig.

In Bezug auf (digitale) Medien finden sich seit Jahrzehnten fachdidaktische Vorschläge zum lernförderlichen Einsatz von Medien in nahezu allen Fachkulturen (z.B. Ladel, Knopf, und Weinberger 2018). Entsprechende didaktische Fragestellungen wurden allerdings nicht von Anfang an aus fachdidaktischen Disziplinen heraus entwickelt, sondern z.T. von zentralen Forschungs- und Entwicklungseinrichtungen (Tulodziecki 2015, 67-68; Kerres 2018, 54).

Die Frage des Beitrages der Fächer zu einer umfassenden Medienbildung, die neben mediendidaktischen auch medienbezogene Erziehungsund Bildungsaufgaben einbezieht, ist hingegen weitaus unsystematischer von den Fachdidaktiken aufgegriffen worden. Im Zuge des gesamtgesellschaftlichen Stellenwerts und der sozialisatorischen Relevanz digitaler Medienentwicklungen scheint sich diese Distanz nun merklich zu verringern. Im Juli 2018 positioniert sich die Gesellschaft für Fachdidaktik (GFD) mit einem Papier zur Fachlichen Bildung in der digitalen Welt (GFD 2018, 1), das die selbsterklärten Aufgaben und Ziele der beteiligten fachdidaktischen Fachgesellschaften in der digital vernetzten Welt beschreibt. Ausgehend vom BMBF-Strategiepapier Bildungsoffensive für die digitale Wissensgesellschaft und der KMK-Strategie Bildung in der digitalen Welt wird insbesondere die fachliche Dimension des Lehrens und Lernens unter den Bedingungen der Digitalisierung fokussiert: 
«Kompetenzerwerb und Bildungsprozesse im Zeichen der Digitalisierung sind untrennbar mit Fachlichkeit und fachlichen Fragen verbunden. Bildung in der digitalen Welt ist deshalb immer auch fachliche Bildung, die im Fachunterricht erfolgt und von den Fachdidaktiken als Wissenschaften des fachlichen Lehrens und Lernens theoretisch, empirisch und konzeptionell in den Blick genommen werden muss.» (ebd., 4-5)

Als die für die Umsetzung zentrale Referenzdisziplin werden explizit die Medienpädagogik genannt (ebd., 2) und vier Ansatzpunkte vorgeschlagen, wie sich fachliche Bildung in der digitalen Welt nun ausschärfen sollte (ebd., 1-3):

1. Fachliche Kompetenzen digital fördern

Die im Fachunterricht aufzubauenden und zu fördernden fachlichen Kompetenzen müssen sich heute in formellen und informellen Kontexten auch «auf digitalen Anwendungs- und Handlungsfeldern bewähren» (ebd., 1). Die Planung fachlicher Lehr- und Lernprozesse muss diese Perspektive einbeziehen und vor dem Hintergrund der Fragen erfolgen, ob sich das fachliche Lernen durch den Einsatz digitaler Medien wirksamer gestalten lässt und ob dieser Einbezug dazu beiträgt, «für die Nutzung fachlicher Kompetenzen in einer digitalen Welt bestmöglich vorzubereiten» (ebd., 2).

2. Digitale Kompetenzen fachlich fördern

Überfachliche Kompetenzbereiche müssen mit fachlichen Perspektiven verbunden werden, denn «[e]rst in der Kombination mit fachlichen Kompetenzen und damit verknüpften Inhalten erhalten digitale Kompetenzen [...] einen Fokus, in dem sie zur Anwendung gelangen und geübt, reflektiert bzw. gefördert werden können» (ebd., 2).

3. Fachliche digitale Kompetenzen über die KMK-Standards hinaus Neben der fachlichen Förderung digitaler Kompetenzen ist der digital vernetzte Wandel auch mit der Entstehung neuer Anforderungen verbunden, die ganz spezifische fachliche digitale Kompetenzen erforderlich machen (ebd., 2-3). So geht etwa die analysierende 
Auseinandersetzung mit schriftsprachlichen Produkten im Fach Deutsch mit der Aufgabe einher, bei der Vermittlung von Sprachkompetenz ganz neue Dimensionierungen zu berücksichtigen, da digitale Erzeugnisse zumeist nicht nur schriftsprachliche, sondern gleichzeitig auch rezeptive und auditive Zugänge aufweisen, die in Kombination zu einem ganz neuen, eigenständigen digitalen Produkt werden und folglich fachlicher digitaler Kompetenzen bedürfen.

4. Digitale personale Bildung im Fachunterricht fördern

Dieser Bereich rekurriert auf eine «fachspezifische Reflexions- und Kritikfähigkeit über digitale Medien» (ebd., 3). Fachgegenstände sollen etwa aus verschiedenen Perspektiven im Hinblick auf ihre Herausforderungen für das Selbst- und Weltverhältnis von Kindern und Jugendlichen beleuchtet werden. Dies kann von der Auseinandersetzung mit sprachlichen, medienkulturgeschichtlichen oder ethischen Aspekten digitaler Kommunikation über die Reflexion über technische Möglichkeiten zum Schutz von digitaler Identität bis hin zu Big Data und Künstlicher Intelligenz reichen.

Die Fachdidaktiken sehen damit ihre Aufgaben im Kontext von Digitalisierung und Mediatisierung als deutlich über den Kompetenzrahmen der KMK hinausgehend. Neben mediendidaktischen Aspekten sollen umfassend Fragen der Medienbildung und das Selbstverständnis des Faches im Kontext von Digitalisierung in den Blick genommen werden. Mit Bezug auf die Medienpädagogik ist dies insofern bemerkenswert, als dass hier ein breites selbstreflexives Arbeitsprogramm formuliert wird, in dem Medien nicht nur eine additive und funktionale Rolle spielen. Für das Fach Deutsch hat Wermke Ende der 1990er Jahre in ähnlicher Weise im Rahmen ihres Ansatzes zur integrativen Medienerziehung auf die Reflexion eines veränderten Selbstverständnisses des Faches in Anbetracht der Medienentwicklung sowie der Veränderung von Gegenstandsbereichen hingewiesen (Wermke 1997, 28-29).

Für das Fach Mathematik findet sich ein erster konzeptioneller Entwurf, der sich der Frage einer umfassenden fachlichen Medienbildung zuwendet (Hischer 2016). Unter dem Begriff des 〈Medialitätsbewusstseins〉 
entfaltet Hischer (ebd., 72) ein Fachverständnis, wonach sich medienpädagogische Zielbereiche nicht als eine zusätzliche Aufgabe verstehen, die an die Fächer herangetragen wird, sondern als unweigerlicher Bestandteil, der zur Erreichung der fachbezogenen Ziele erforderlich ist und immer schon war. Diese Auffassung steht in einem engen Zusammenhang mit einem medienpädagogischen Grundverständnis, wonach die Reflexion über und der Einsatz von Medien immanenter Bestandteil eines jeden Unterrichts sein müssen, weil Medien Werkzeuge der Weltaneignung sind und damit immer einen Bezug zu Fachfragen haben (Wagner 2013, 60-61). Medialitätsbewusstsein im Mathematikunterricht bedeutet, (digitale) Medien hinsichtlich ihrer Relevanz für mathematische Prozesse in den Blick zu nehmen. Medien sollen nicht nur zur Anwendung von, sondern (immer) auch zur «Reflexion über Mathematik» (Hischer 2016, 409) genutzt werden. So lassen sich etwa soziale Netzwerke als charakteristische Onlinedienste der Digitalisierung im Mathematikunterricht sowohl unter graphentheoretischen Modellierungsaspekten als auch aus der Perspektive sozialer Prozesse thematisieren, mit dem Ziel, so einen umfassenden fachlichen Beitrag zur Medienbildung zu leisten (ebd., 405-406).

\section{Fazit}

Eine umfassende schulische Medienbildung im Sinne des Lernens mit und über Medien wird aus der Disziplin der Medienpädagogik heraus begründet. Sie weist - insbesondere angesichts der alle Lebensbereiche umfassenden Digitalisierung und Mediatisierung - vielfältige Verbindungen zu Bezugsdisziplinen und Unterrichtsfächern auf. Insofern sind medienpädagogische Inhaltsbereiche auch zentrale Gegenstände fachdidaktischer Disziplinen. Darüber hinaus führen Entwicklungen im Bereich der Digitalisierung auch zu neuen Gegenständen und Methoden einzelner Fächer, die wieder Rückwirkungen auf allgemeinbildende (Medien-)Kompetenzen haben können. 


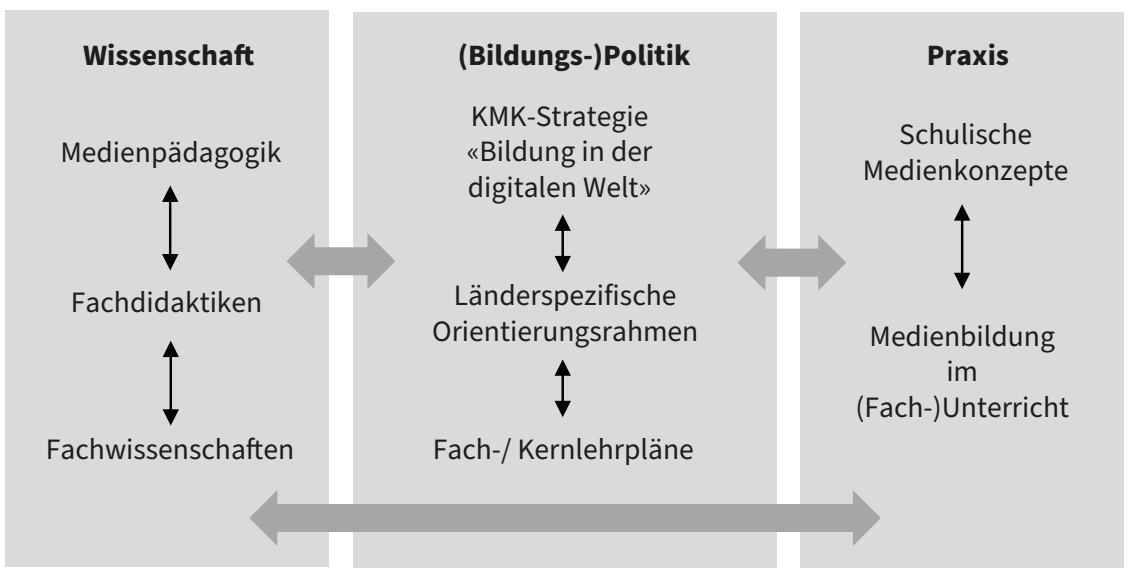

Abb. 1.: Ebenen und Spannungsfelder schulischer Medienbildung.

Setzt man bildungspolitisch auf eine integrative Realisierung von $\mathrm{Me}$ dienbildung, d.h. ohne einen eigenen Lernbereich oder ein eigenes Fach, so bedeutet dies, dass entsprechend abgestimmte schulische Lerngelegenheiten geschaffen werden müssen, die in der Summe der Beiträge aller Unterrichtsfächer einen umfassenden Kompetenzerwerb ermöglichen.

Neben der Entwicklung von - auf die KMK-Strategie abgestimmten - länderspezifischen Orientierungsrahmen und medienbezogenen Fachbzw. Kernlehrplänen besteht derzeit die Herausforderung in der Entwicklung fachdidaktischer Zugänge zur Medienbildung und deren Vernetzung unter einem medienpädagogisch begründeten Orientierungsrahmen zur Medienbildung. Da es dabei nicht nur um die konzeptionelle curriculare Anbindung gehen kann, sondern auch um forschungsbasierte Zugänge, ist dies ein langfristiger Prozess, der auch viele Anschluss- und Verbindungsmöglichkeiten zu medienpädagogischer Forschung im Schulkontext bietet. Angesichts der mit der KMK-Strategie verbundenen engen zeitlichen Vorgaben kann jedoch nicht in allen Fächern davon ausgegangen werden, dass die bildungspolitischen Ordnungsdokumente auch forschungsbasiert sind, sondern zunächst noch stärker normative Züge tragen.

Die angemessene und erfolgreiche Umsetzung der mit der KMK-Strategie verbundenen Ziele in der dort vorgeschlagenen integrativen Variante hängt also insbesondere davon ab, inwieweit die genannten fachdidaktischen Aufgaben erfolgreich bewältigt und umgesetzt werden können. Darüber hinaus muss sich dies in hochschulischer Ausbildung von Lehrkräften 
und in der Lehrerinnen- und Lehrerfortbildung niederschlagen, damit auf der Basis schulischer Medienkonzepte dann in entsprechendem fachbezogenen Unterricht Lernaktivitäten zur Medienbildung initiiert, unterstützt, begleitet und reflektiert werden können. Dabei wird auch zu prüfen sein, ob die integrative Strategie realistisch ist oder ob nicht eine Verbindung von einem verpflichtenden fachübergreifenden Lernbereich, z.B. in der Sekundarstufe I, und fachbezogenen Zugängen zur Medienbildung die zielführendere Alternative ist. Eine solche Perspektive könnte sich auch dann aufdrängen, wenn es nicht gelingt, den Anspruch einer Medienbildung in Bezug auf zentrale Ziel- und Inhaltsbereiche allein durch fachdidaktische Beiträge zu realisieren.

Auch die Sicherstellung der informatischen Anteile der Medienbildung ist angesichts des Lehrkräftemangels in diesem Bereich und der fehlenden Verankerung als Pflichtfach noch ungelöst.

\section{Literatur}

Abraham, Ulf, und Martin Rothgangel. 2017. «Fachdidaktik im Spannungsfeld von 〈Bildungswissenschaft〉 und 〈Fachwissenschaft〉》. In Allgemeine Fachdidaktik, herausgegeben von Horst Bayrhuber, Ulf Abraham, Volker Frederking, Werner Jank, Martin Rothgangel, Helmut J. Vollmer, und Horst Bayrhuber, 15-21. Auf dem Weg zu einer Allgemeinen Fachdidaktik, Horst Bayrhuber, Ulf Abraham, Volker Frederking, Werner Jank, Martin Rothgangel, Helmut Johannes Vollmer; Band 1. Münster New York: Waxmann.

Bayrhuber, Horst. 2017. «Aufgaben der Allgemeinen Fachdidaktik». In Allgemeine Fachdidaktik, herausgegeben von Horst Bayrhuber, Ulf Abraham, Volker Frederking, Werner Jank, Martin Rothgangel, Helmut J. Vollmer, und Horst Bayrhuber, 248-53. Auf dem Weg zu einer Allgemeinen Fachdidaktik, Horst Bayrhuber, Ulf Abraham, Volker Frederking, Werner Jank, Martin Rothgangel, Helmut Johannes Vollmer ; Band 1. Münster New York: Waxmann.

Comenius, J.A. 1658. Orbis sensualium pictus. Nachdruck der Erstausgabe von 1658. Dortmund: Harenberg.

GFD [Gesellschaft für Fachdidaktik e.V.]. 2018. «Fachliche Bildung in der digitalen Welt. Positionspapier der Gesellschaft für Fachdidaktik». http://www.fachdidaktik.org/wp-content/uploads/2018/o7/GFD-Positionspapier-Fachliche-Bildung-in-der-digitalen-Welt-2018-FINAL-HP-Version.pdf.

Heimann, Paul. 1962. «Didaktik als Theorie und Lehre», Deutsche Schule, 54 (9): 407-27. 
Herzig, Bardo. 2012. Medienbildung. Grundlagen und Anwendungen. Bd. 1. Handbuch Medienpädagogik. München: kopaed.

Hischer, Horst. 2016. Mathematik - Medien - Bildung. Medialitätsbewusstsein als Bildungsziel: Theorie und Beispiele. Wiesbaden: Springer Fachmedien Wiesbaden. https://doi.org/10.1007/978-3-658-14167-7_1.

Kerres, Michael. 2018. Mediendidaktik: Konzeption und Entwicklung digitaler Lernangebote. 5. Aufl. De Gruyter Studium. Berlin: Walter de Gruyter GmbH.

KMK [Kultusministerkonferenz]. 1995. «Medienpädagogik in der Schule. Erklärung vom 12.05.1995». Berlin: Sekretariat der KMK.

KMK [Kultusministerkonferenz]. 2016. «Bildung in der digitalen Welt. Strategie der Kultusministerkonferenz. Beschluss vom 08.12.2016». Berlin: Sekretariat der KMK.

KVFF [Konferenz der Vorsitzenden Fachdidaktischer Fachgesellschaften]. 1998. «Fachdidaktik in Forschung und Lehre». Kiel: Institut für Pädagogik der Naturwissenschaften. http://www.fachdidaktik.org.

Ladel, Silke, Julia Knopf, und Armin Weinberger, Hrsg. 2018. Digitalisierung und Bildung. Wiesbaden: Springer Fachmedien Wiesbaden. https://doi. org/10.1007/978-3-658-18333-2.

Leuders, Timo. 2015. «Empirische Forschung in der Fachdidaktik. Eine Herausforderung für die Professionalisierung und die Nachwuchsqualifizierung». Beiträge zur Lehrerinnen- und Lehrerbildung 33 (2): 215-34. http://nbn-resolving. org/urn:nbn:de:0111-pedocs-138860.

Parchmann, Ilka. 2013. «Wissenschaft Fachdidaktik - Eine besondere Herausforderung». Beiträge zur Lehrerinnen- und Lehrerbildung 31 (1): 31-41.

Sektion Medienpädagogik der Deutschen Gesellschaft für Erziehungswissenschaft - DGfE. 2018. «Orientierungsrahmen für die Entwicklung von Curricula für medienpädagogische Studiengänge und Studienanteile». In Futurelab Medienpädagogik: Qualitätsentwicklung, Professionalisierung, Standards. Thesenpapier zum Forum Kommunikationskultur 2017 der GMK, herausgegeben von Thomas Knaus, Dorothee M. Meister, und Kristin Narr, 201-9. Schriften zur Medienpädagogik 54. München: kopaed. https://doi.org/10.21240/ mpaed/00/2017.12.04.X.

Spanhel, Dieter. 1999. Integrative Medienerziehung in der Hauptschule: ein Entwicklungsprojekt auf der Grundlage responsiver Evaluation. München: KoPäd-Verl.

Tulodziecki, Gerhard. 2015. «Medienpädagogik von der Nachkriegszeit bis zum Ende der 1970er-Jahre». In Die Geschichte der Medienpädagogik in Deutschland, herausgegeben von Friederike von Gross, Dorothee Meister, und Uwe Sander, 63-93. Weinheim: Beltz Juventa.

Tulodziecki, Gerhard, Bardo Herzig, und Silke Grafe. 2019. Medienbildung in Schule und Unterricht: Grundlagen und Beispiele. 2. Aufl. Bad Heilbrunn: Verlag Julius Klinkhardt. 
Tulodziecki, Gerhard, und Bardo Herzig. 2009. Handbuch Medienpädagogik, Bd. 2: Mediendidaktik: Medien in Lehr- und Lernprozessen verwenden. München: kopaed.

Vollmer, Helmut J. 2017. «Zur jüngeren Entwicklung der Fachdidaktiken in Deutschland». In Allgemeine Fachdidaktik, herausgegeben von Horst Bayrhuber, Ulf Abraham, Volker Frederking, Werner Jank, Martin Rothgangel, Helmut J. Vollmer, und Horst Bayrhuber, 11-14. Auf dem Weg zu einer Allgemeinen Fachdidaktik, Horst Bayrhuber, Ulf Abraham, Volker Frederking, Werner Jank, Martin Rothgangel, Helmut Johannes Vollmer ; Band 1. Münster New York: Waxmann.

Wagner, Wolf-Rüdiger. 2013. Bildungsziel Medialitätsbewusstsein: Einladung zum Perspektivwechsel in der Medienbildung. München: kopaed.

Weißeno, Georg. 2015. «Konstruktion einer politikdidaktischen Theorie». In Empirische Forschung in gesellschaftswissenschaftlichen Fachdidaktiken, herausgegeben von Georg Weißeno und Carla Schelle, 3-20. Wiesbaden: Springer Fachmedien Wiesbaden. https://doi.org/10.1007/978-3-658-06191-3_1.

Wermke, Jutta. 1997. Integrierte Medienerziehung im Fachunterricht: Schwerpunkt: Deutsch. München: KoPäd-Verl.

Wilkens, Ulrike. 2000. Das allmähliche Verschwinden der informationstechnischen Grundbildung: zum Verhältnis von Informatik und Allgemeinbildung. Als Ms. gedr. Berichte aus der Informatik. Aachen: Shaker. 